\title{
Ocurrencia de escuticociliatosis en el lenguado Paralichthys adspersus causado por Miamiensis avidus, en Perú
}

\section{Occurrence of scuticociliatosis in the flounder Paralichthys adspersus caused by Miamiensis avidus, in Peru}

\section{Marco Medina $^{1 *}$, Giovanna Sotil ${ }^{2}$, Violeta Flores ${ }^{1}$ y Carla Fernandez ${ }^{1}$}

\author{
1 Laboratorio de Patobiología Acuática, Centro de Investigaciones Acuícolas Alexander Von Humboldt, Instituto del Mar del Perú \\ 2 Laboratorio de Genética Molecular, Centro de Investigaciones Acuícolas Alexander Von Humboldt, Instituto del Mar del Perú. \\ Dirección Postal: IMARPE, Esquina Gamarra y General Valle S/N Chucuito, Callao, Lima. Perú \\ Casilla Postal: callao 01 \\ *Autor para correspondencia \\ Email Marco Medina: mmedina@imarpe.gob.pe \\ Email Giovanna Sotil: gsotil@imarpe.gob.pe \\ ORCID Giovanna Sotil: http://orcid.org/0000-0002-1583-8821 \\ Email Violeta Flores: vflores@imarpe.gob.pe \\ Email Carla Fernández: cafernandez@imarpe.gob.pe
}

\section{Resumen}

\begin{abstract}
La escuticociliatosis es una enfermedad causada por ciliados del orden Scuticociliatida los cuales se caracterizan por su alto potencial para invadir al huésped, significando un serio problema en la acuicultura marina. El presente trabajo describe la infección con escuticociliados en lenguado Paralichthys adspersus en cautiverio. Los signos externos e internos de la infección incluyen zonas necróticas en el tegumento, abundante mucosidad, abultamiento de la cavidad visceral con acumulación de líquido ascítico, necrosis de las fibras musculares, licuefacción del cerebro, entre otros. Se identificó y caracterizó molecularmente al parásito ciliado como $M$. avidus, utilizando secuencias de gen mitocondrial citocromo oxidasa I (COI), y de los genes nucleares $\beta$-tubulina y la región de la subunidad menor rRNA 18S, mostrando una sinonimia con $P$. dicentrarchi. Todas las lesiones estuvieron invadidas de ciliados. A nivel histológico, se detectaron ciliados en casi todos los tejidos siendo el cerebro el órgano más parasitado. Los ejemplares infectados, acompañados de infecciones bacterianas secundarias predominantemente por Vibrio alginolyticus, después de un periodo de letargia mueren.
\end{abstract}

Palabras clave: escuticociliatosis; Miamiensis avidus; lenguado Paralichthys adpsersus; acuicultura; parásitos ciliados.

\section{Abstract}

The Scuticociliatosis, a disease caused by ciliates from the order Scuticociliatida characterized by their high potential to invade the host, is a serious problem in marine aquaculture. This paper describes scuticociliatosis infection in farmed flounder Paralichthys adspersus. External and internal signs of infection include necrotic areas in the tegument, abundant mucus, swelling of the visceral cavity with ascitic fluid accumulation, necrotic muscle fibers and brain liquefaction, among others. The ciliate parasite was molecularly identified and characterized as M. avidus, using sequences of mitochondrial gene cytochrome oxidase I (COI), and nuclear genes $\beta$-tubulin and the region of the small subunit $18 \mathrm{~S}$ rRNA, showing synonymy with P. dicentrarchi . All lesions were infested by ciliates. Histologically, ciliates are detected in almost all tissues being the brain the organ more parasitized. The infected specimens associated with secondary bacterial infections, predominantly Vibrio alginolyticus, died after a lethargy period.

Keywords: scuticociliatosis; Miamiensis avidus; flounder; Paralichthys adpsersus; aquaculture; ciliate parasite.

Citación:

Medina M., G. Sotil, V. Flores y C. Fernandez. 2016. Ocurrencia de escuticociliatosis en el lenguado Paralichthys adspersus causado por Miamiensis avidus, en Perú. Revista peruana de biología 23(3): 261 270 (Diciembre 2016). doi: http://dx.doi.org/10.15381/rpb.v23i3.12861
Fuentes de financiamiento:

La presente investigación fue financiada por el Programa Nacional de Innovación para la Competitividad y productividad INNÓVATE-PERÚ bajo el Proyecto Convenio N²36-FINCyT-IA 2013 "Producción de semilla del lenguado Paralichthys adspersus en cautiverio: II Mejoramiento de las técnicas de larvicultura".

\section{Información sobre los autores:}

MM y GS diseñaron el estudio, MM colectó las muestras, realizó la descripción histológica, GS realizó el análisis de identificación molecular, VF y CF realizaron el análisis microbiológico y MM, GS, VF y CF redactaron y aprobaron el manuscrito

Los autores no incurren en conflictos de intereses.

Journal home page: http://revistasinvestigacion.unmsm.edu.pe/index.php/rpb/index

(c) Los autores. Este artículo es publicado por la Revista Peruana de Biología de la Facultad de Ciencias Biológicas, Universidad Nacional Mayor de San Marcos. Este es un artículo de acceso abierto, distribuido bajo los términos de la Licencia Creative Commons Atribución-NoComercial-Compartirlgual 4.0 Internacional.(http://creativecommons.org/licenses/by-nc-sa/4.0/), que permite el uso no comercial, distribución y reproducción en cualquier medio, siempre que la obra original sea debidamente citadas. Para uso comercial, por favor póngase en contacto con editor.revperubiol@gmail.com. 


\section{Introducción}

El lenguado Paralichthys adspersus (Steindachner 1867) es una especie de pez plano con alto valor comercial, que se distribuye desde Paita en Perú hasta las islas Juan Fernández en Chile (Pequeño 1989, Acuña \& Cid 1995, Siefeld et al. 2003). En el Perú, es una especie relativamente nueva para cultivo y por lo tanto se conoce poco de las enfermedades a las que es propensa en cautiverio. Constituye una de las especies priorizadas para diversificar la acuicultura marina (PRODUCE 2013), cultivada tanto con fines de investigación (en Instituto del Mar del Perú y el Fondo Nacional de Desarrollo Pesquero, FONDEPES) como a escala piloto comercial en la costa centro del Perú, no estando ajenos a las enfermedades que enfrenta la acuicultura a nivel mundial.

Un serio problema en la acuicultura marina es la escuticociliatosis, debido a las altas tasas de mortalidad a las que está asociado. Esta enfermedad es causada por ciliados histiófagos del orden Scuticociliatida de vida libre, que son parásitos facultativos. Son considerados carrońeros; bajo ciertas circunstancias se comportan como parásitos histiófagos oportunistas, que se alimentan de material orgánico en suspensión así como de células y restos de tejidos de organismos acuáticos (Elston et al. 1999, Moustafa et al. 2010). Se han descrito numerosas especies que se caracterizan por su alto potencial para invadir al huésped, entre los que destacan Uronema marinum (Dujardin 1841), Philastherides dicentrarchi (Dagresco et al. 1995) y Miamiensis avidus (Thompson \& Moewus 1964) que han sido reportados por causar mortalidades en peces como en el lenguado japonés P. olivaceus (Yoshinaga \& Nakazoe 1993; Jee et al. 2001, Kim et al. 2004), el rodaballo Scophthalmus maximus (Dyková \& Figueras 1994, Sterud et al. 2000, Iglesias et al. 2001), la lubina Dicentrarchus labrax (Dragesco et al. 1995), el atún Thunnus maccoyii (Munday et al. 1997), la palometa Pampus argentus (Al-Marzouk \& Azad 2007, Azad et al. 2007), el tiburón Stegostoma fasciatum (Stidwothy et al. 2013), el caballito de mar Hippocampus erectus (Thompson \& Moewus 1964) y dragones de mar Phycodurus eques y P. taeniolatus (Rossteuscher et al. 2008), con consecuencias económicas significativas (Scholz 1999, Piazzon et al. 2013)

Algunos reportes mencionan que la posible infección primaria de estos parásitos ocurre principalmente por las branquias y piel (Paraná et al. 2003), a partir de donde ingresan a los órganos internos vía el torrente sanguíneo. Los peces afectados secretan excesivo mucus corporal y evidencian daño dermal, incluyendo lesiones superficiales tales como áreas blanquecinas y tejido necrótico (Jin et al. 2009, Harikrishnan et al. 2010, Moustafa et al. 2010). La enfermedad está asociada con varios cambios patológicos incluido sangrado, úlceras cutáneas, distrofia y necrosis muscular, anemia hipocrómica y encefalitis asociada con ablandamiento y licuefacción del tejido cerebral (Iglesias et al. 2001).

En la actualidad, no existe un tratamiento efectivo para la escuticociliatosis. Algunos métodos consideran baños con formalina que particularmente pueden eliminar los parásitos mientras permanecen fuera del hospedero, pero que es ineficaz una vez que los ciliados se alojan al interior del pez (Iglesias et al. 2002). Es por esto que resulta necesario la identificación de los agentes patógenos que conducen a enfermedades en esta especie y los daños que ocasionan, con el objetivo de definir protocolos para su detección y tratamiento oportuno (Silva 2010), sin embargo debido a los diferentes criterios que se han utilizado para su discriminación, la identificación de estos organismos es actualmente bastante confusa (Budiño Rodríguez 2011).

Muchos estudios taxonómicos y reconstrucciones filogenéticas de diferentes ciliados han sido desarrollados utilizando aspectos de su morfología externa, comportamiento, capacidad epidemiológica, etc. (Kim et al. 2004, Jung et al. 2005, Song et al. 2009, Song et al. 2013), sin embargo se ha reportado que muchas cepas pueden presentar características externas similares pero con diferencias a nivel molecular (Liu et al. 2013), tanto intra como interespecíficas. Los estudios moleculares vienen contribuyendo de forma importante en la discriminación de especies dentro los Ciliophora, pero el uso de un solo marcador puede ser cuestionado debido a las limitaciones que presentan para los estudios evolutivos, dadas las variaciones genéticas existentes entre ciliados ( $\mathrm{Li}$ et al. 2013). Así por ejemplo, el análisis de la subunidad menor del ARN ribosomal (SSU 18S) ha permitido realizar una diferenciación interespecífica de ciliados (Hillis \& Dixon 1991) debido a alto grado de conservación que presenta, mayor longitud de comparación y disponibilidad de datos con que se cuenta para este grupo, en comparación con otros marcadores. Por otro lado, el gen mitocondrial citocromo oxidasa I (COI) propuesto como un buen marcador para la identificación de especies de animales a través del uso del código de barras de ADN (Herbert et al. 2003), parece estar asociado a la identificación de variaciones intraespecíficas en estos ciliados (Jung et al. 2011).

El presente trabajo reporta por primera vez en el Perú la ocurrencia de escuticociliatosis ocasionado por el protozoario ciliado $M$. avidus (sinónimo $P$. dicentrarchi), como el agente causal responsable de la mortalidad, en este estudio, del lenguado nativo $P$ adspersus mantenido en cautiverio. El parásito ha sido identificado molecularmente, considerando además las descripciones de los signos clínicos externos y daños en órganos internos ocasionados por la infección.

\section{Materiales y métodos}

Colecta de muestras infectadas.- Se realizó el estudio de ejemplares moribundos de Paralichthys adspersus, criados en cautiverio, durante un brote de escuticociliatosis en enero del 2014 procedentes de un centro de cultivo de la costa central de Ancash, Perú. El brote causo una mortalidad de alrededor del $70 \%$. Los ejemplares estudiados tuvieron un peso húmedo entre 10 a $400 \mathrm{~g}$ y fueron examinados considerando aspectos externos (como pigmentación, hemorragias, abultamiento del cuerpo) e internos (hemorragias, mucosidad, necrosis, etc). Se tomaron muestras en fresco de branquias, líquido ascítico y mucosa epitelial, los que fueron evaluados preliminarmente utilizando un microscopio compuesto para corroborar la presencia de parásitos.

Aislamiento de ciliados y bacterias a partir de las lesiones.Los escuticociliados fueron aislados del líquido colectado por abdominocentesis de la cavidad visceral utilizando una jeringa de $1 \mathrm{~mL}$. El líquido extraído fue colocado en medio Leibovitz 1-15 (Sigma Aldrich ${ }^{\circledast}$ Israel), al cual se le agregó suero fetal bovino FBS (Gibco ${ }^{\oplus}$ EEUU) y solución antibiótica antimicótica PSA (Gibco ${ }^{\oplus}$, EEUU) conteniendo 100 U. $\mathrm{mL}^{-1}$ de penicilina, 100 $\mu \mathrm{g} \cdot \mathrm{mL}^{-1}$ de estreptomicina y $0.25 \mu \mathrm{g} \cdot \mathrm{mL}^{-1}$ de anfotericina $\mathrm{B}, \mathrm{y}$ colocado en incubación a $22{ }^{\circ} \mathrm{C}$ (Iglesias et al. 2003). La cepa fue denominada como 1PERU. 
Para el aislamiento de bacterias, se tomaron muestras de bazo y riñón asépticamente y se colocaron en caldo infusión cerebro corazón (BHI broth, Oxoid ${ }^{\oplus}$, Inglaterra) para posteriormente estrialos sobre placas con agar tiosulfato citrato bilis sacarosa (Oxoid $^{\oplus}$, Inglaterra) y tripticasa de soya suplementado con cloruro de sodio al 1\% (MERCK ${ }^{\oplus}$, Alemania). Luego de 48 horas, se evaluaron las placas utilizando el sistema de identificación bacteriana API 20 E (Biomeriux ${ }^{\oplus}$, Francia).

Identificación molecular.- Un cultivo in vitro de la cepa 1PERU fue centrifugado a $10000 \times \mathrm{g}$ por $20 \mathrm{~min}$ para concentrar la muestra de ciliados y realizar la extracción de ADN por el método de CTAB2X. Se seleccionaron tres marcadores para su identificación, el gen mitocondrial citocromo oxidasa I (COI), y los genes nucleares $\beta$-tubulina y la subunidad menor rRNA 18S. La PCR se realizó en un termociclador Proflex (Applied Biosystems-Thermo Scientific), utilizando $10 \mathrm{ng}$ de ADN, $2.5 \mathrm{U}$ de Taq polimerasa HotStart (Qiagen, Switzerland), $0.1 \mu \mathrm{M}$ de cebadores SSU1-F AACCTGGTTGATCCTGCCAGT / SSU2-R TGATCCTTCTGCAGGTTCACCTAC (Medlin et al. 1988) para la amplificación del 18S, FCO1 TCAGGTGCTGCACTAGC / RCOX1 TAAACTTCAGGGTGACCAAAAAT (Lynn \& Strüder-Kypke 2006) para COI, y FPTubin CCTACCACGGAGACTCTGATT / RPTubin CCATAATTCTGTCGGGGTATT (Budiño et al. 2011, Leiro et al. 2000) para $\beta$-tubulina, en una reacción de $10 \mu \mathrm{L}$ de volumen final. Las temperaturas de hibridación utilizadas en la PCR fueron de $52^{\circ} \mathrm{C}(18 \mathrm{~S}), 50^{\circ} \mathrm{C}(\mathrm{COI})$ y $57^{\circ} \mathrm{C}$ ( $\beta$-tubulina). Los productos fueron evaluados en agarosa $1 \%$, purificados con el kit PCR purification (Bioneer), y secuenciados para ambas direcciones en un analizador genético $\mathrm{ABI}$ 3730XL (Applied Biosystems - Thermo Scientific) utilizando los mismos cebadores de la amplificación.

Las secuencias de ambas hebras fueron editadas visualmente con el programa Chromas Lite versión 2.1 (Technelysium Pty Ltd, South Brisbane, Australia). La identificación molecular se realizó utilizando la herramienta BLASTn del NCBI, el alineamiento y consenso de secuencias se realizó con el software Clustal W (Thompson et al. 1994) y la edición con el programa BioEdit. Los árboles filogenéticos fueron construidos con el programa MEGA v6 (Tamura et al. 2013) utilizando el método Neighbour-Joining (NJ) y el modelo de distancia Kimura2-parámetros $(\mathrm{K} 2 \mathrm{P})$, considerando 1000 réplicas. Los estimados de divergencia fueron calculados utilizando el modelo K2P y la comparación se realizó incluyendo otras secuencias nucleotídicas del Phylum Ciliophora, debido el limitado número de secuencias disponibles para el género en estudio.

Microscopía electrónica de barrido (SEM).- Los ciliados fueron concentrados por centrifugación a $600 \times \mathrm{g}$ por $5 \mathrm{~min}$ a partir de cultivos in vitro, para posteriormente realizar la fijación del precipitado en una solución de glutaraldehído en buffer fosfato $0.1 \mathrm{M}$, postfijado en tetraóxido de osmio $2 \%$, deshidratado en una gradiente decreciente de concentraciones de etanol, secado a punto crítico, metalizados con oro y examinado en un microscopio electrónico de barrido Fei Inspect S50.

Histología.- Los órganos y tejidos seleccionados fueron inmediatamente fijados en formalina tamponada al 10\%, y posteriormente deshidratados utilizando un procesador automático de tejidos Leica ${ }^{\oplus}$. Las muestras fueron embebidas en parafina a partir de los cuales se realizaron cortes histológicos de $4 \mu \mathrm{m}$ con un micrótomo Leica ${ }^{\oplus}$, coloreados con hematoxilina y eosina $(\mathrm{H} \& \mathrm{E})$ y fotografiados en un microscopio Nikon ${ }^{\circledR} 90$ eclipse.

\section{Resultados}

Descripción de signos clínicos externos e internos en P. adspersus con escuticociliatosis.- Externamente, los peces afectados mostraron alteraciones en la natación, hiperpigmentación en el lado dorsal, despigmentación difusa sobre el dorso y en la base del opérculo (Fig. 1A, B), aletas rasgadas hemorrágicas, abdomen abultado (Fig. 1C) y prolapso del intestino. Internamente los signos clínicos más representativos fueron la acumulación de líquido ascítico, hígado agrandado, intestino hemorrágico e inflamado (Fig. 1D).

La despigmentación sobre el dorso de los peces se observó a modo de manchas grises y difusas, con lesiones escamosas, abundante mucosidad y restos de tejido necrosado. Además, el líquido ascítico y cerebroespinal coincidió con la presencia de un gran número de ciliados. No se evidenció exoftalmia ni inflamación periorbital.

Aislamiento de bacterias oportunistas.- Adicional a la infección por protozoarios ciliados, la siembra de muestras de órganos internos como bazo, riñón y raspados de piel propiciaron el crecimiento de colonias amarillas, mucosas de borde irregular sobre agar TCBS las que fueron identificadas como Vibrio alginolyticus, especie predominante en los cultivos microbiológicos.

Identificación molecular.- Se obtuvieron secuencias nucleotídicas con tamaños de 1581 pb para el gen 18S, 724 pb para COI, y $360 \mathrm{pb}$ para $\beta$-tubulina, las que fueron depositadas en el GenBank con los números de accesión KX259258 a KX259260, respectivamente. El análisis con los 3 marcadores en conjunto permitieron ubicar a la cepa 1PERU dentro de la Familia Philasteridae, identificándolo como Miamiensis avidus (Thompson \& Moewus 1964) sinónimo con Philastherides dicentrarchi, y discriminándolo del género Philasterides con el marcador 18S (Fig. 2).

La secuencia de la región parcial de la subunidad menor rRNA $18 \mathrm{~S}$ analizada mostró una identidad del $100 \%$ con $P$. dicentrarchi JX914665 y $M$. avidus JN689230, un 95\% con $P$. armatalis FJ848877, y 94\% con Philaster apodigitiformis FJ648350. No se registraron sustituciones entre secuencias de la cepa 1PERU y las secuencias obtenidas del GenBank, mientras que comparando con $P$. armatalis se observaron 75 sustituciones. La cepa 1 PERU no mostró divergencias con $M$. avidus ni $P$. dicentrarchi, pero sí con $P$. armatalis (0.049, Tabla 1). A partir del análisis de agrupamiento, se observó la formación de un solo clado con M. avidus (Thompson \& Moewus 1964) y su discriminación respecto a los géneros Philasterides (Kahl 1931) representado por P. armatalis, (Song 2000) y Philaster (Fabre-Domergue 1885) representado por $P$. apodigitiformis (Miao 2009), por lo que se corrobora su sinonimia con $P$. dicentrarchi.

El análisis realizado de la cepa 1PERU con la secuencia COI mostró un 99\% de identidad con $M$. avidus EU831233, 93\% con $P$. dicentrarchi GQ342957, y un $80 \%$ con Tetrabymena sp. EF070320 y Cyclidium sp. FJ905128. Se identificaron 47 sustituciones nucleotídicas al compararla con $M$. avidus, y 79 sustituciones cuando se incluye a $P$. dicentrarchi. Las estimaciones de divergencias estuvieron entre $0.001-0.066$ para la misma especie, siendo mayores a 0.2 respecto a otros géneros (Tabla 2). El árbol de distancias basado en el análisis NJ agrupó en un solo 

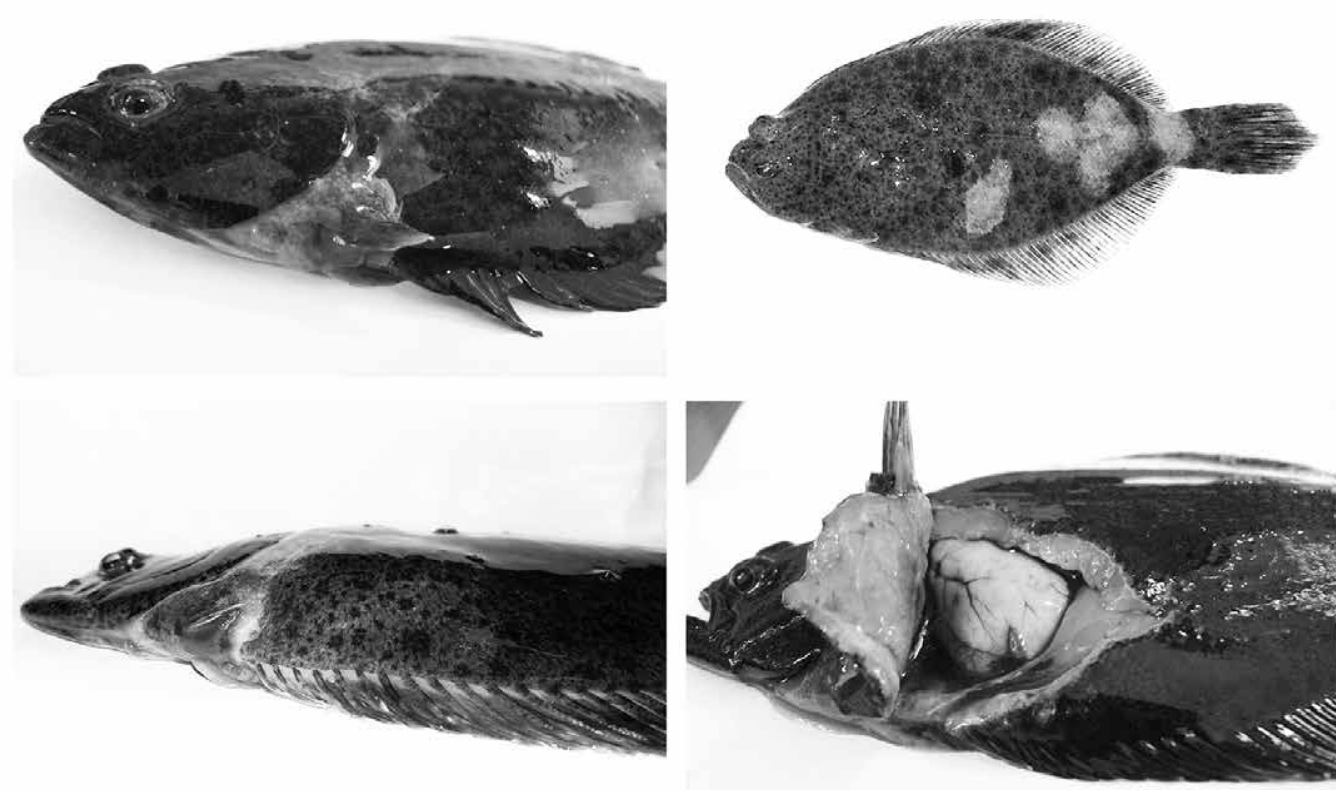

Figura 1. Signos clínicos externos e internos del lenguado $P$. adspersus con escuticociliatosis. Se aprecia despigmentación en la zona abdominal $(A)$, despigmentación en la zona dorsal a modo de manchas blancas difusas (B), abultamiento del área visceral producida por acumulación de líquido ascítico e inflamación intestinal (C) hígado agrandado, intestino inflamado y acumulación de líquido ascítico en la cavidad peritoneal(D).

(A)

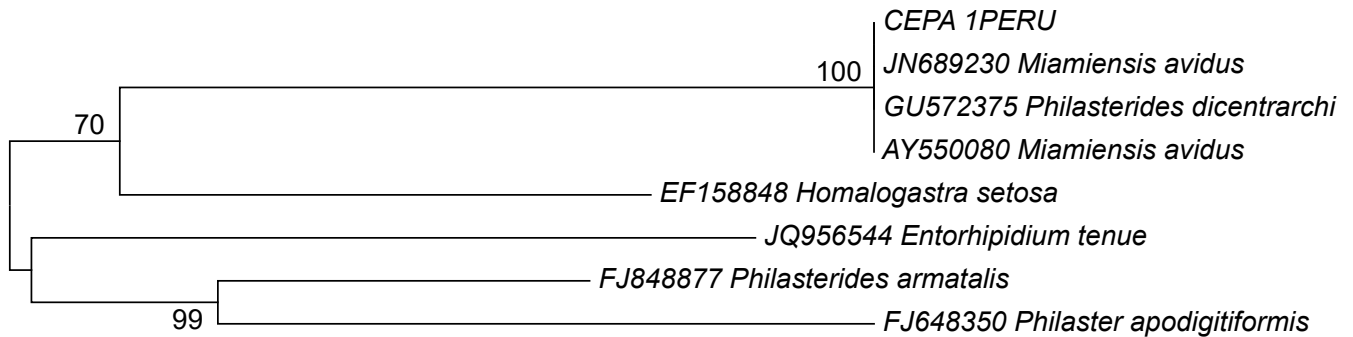

(B)

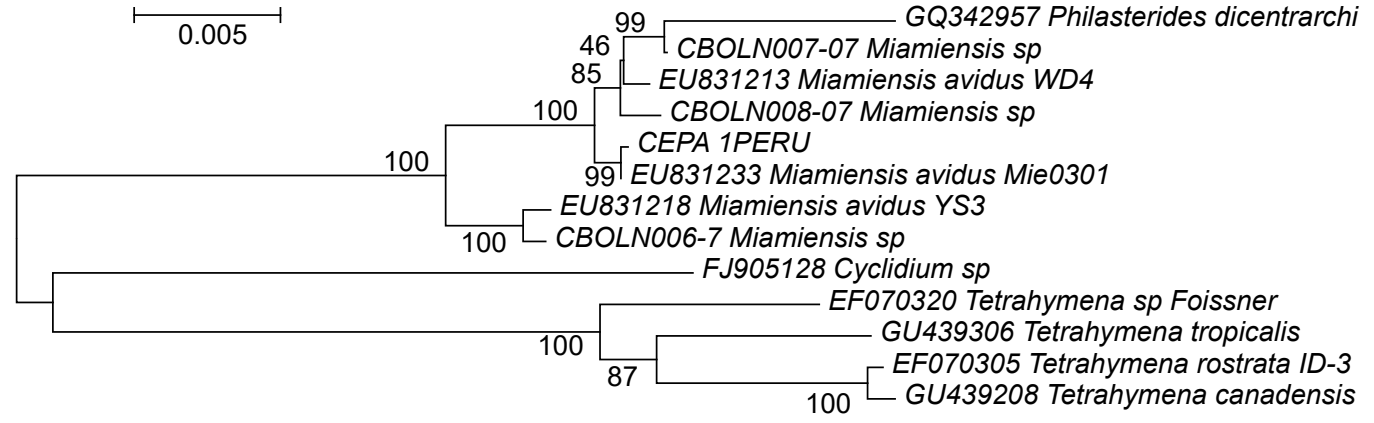

(C)

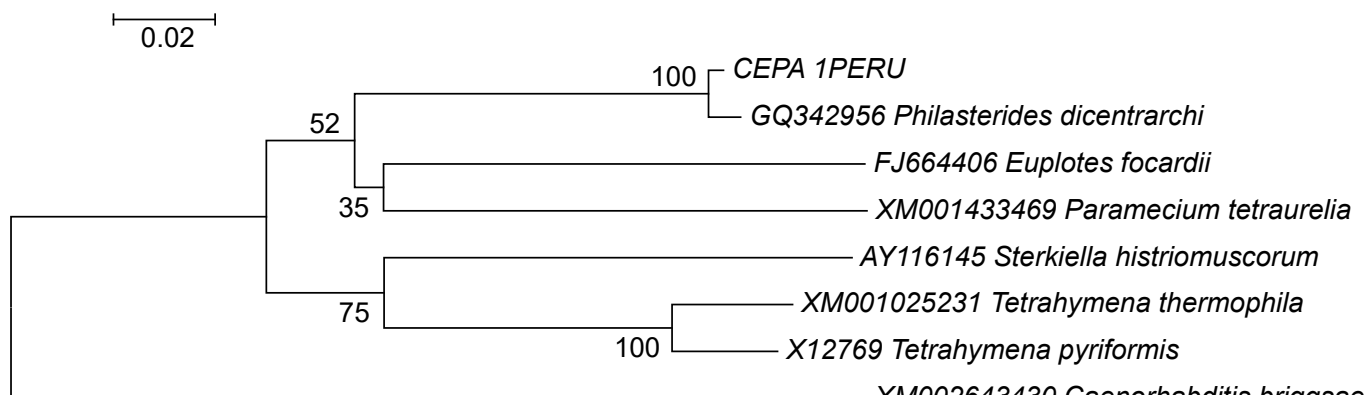

$$
\longmapsto .02
$$

Figura 2. Relaciones filogenéticas de la cepa aislada 1PERU respecto a diversas especies del Phylum Ciliophora, inferido por el análisis de secuencias de los genes rRNA18S (A), COI (B) y $\beta$-tubulina (C), utilizando el método de agrupamiento NJ. El valor de bootstrap para 1000 réplicas está indicado en cada nodo. 
Tabla 1. Estimaciones de divergencia entre secuencias comparadas utilizando el marcador 18S, basado en el número de sustituciones por sitio entre secuencias, con el modelo K2P.

\begin{tabular}{|c|c|c|c|c|c|c|c|}
\hline Cepa (Número de accesión) & 1 & 2 & 3 & 4 & 5 & 6 & 7 \\
\hline 1. CEPA 1PERU & & & & & & & \\
\hline 2. Miamiensis avidus (JN689230) & 0.000 & & & & & & \\
\hline 3. Miamiensis avidus (AY550080) & 0.000 & 0.000 & & & & & \\
\hline 4. Philasterides dicentrarchi (GU572375) & 0.000 & 0.000 & 0.000 & & & & \\
\hline 5. Philasterides armatalis (FJ848877) & 0.049 & 0.049 & 0.049 & 0.049 & & & \\
\hline 6. Philaster apodigitiformis (FJ648350) & 0.057 & 0.057 & 0.057 & 0.057 & 0.035 & & \\
\hline 7. Homalogastra setosa (EF158848) & 0.044 & 0.044 & 0.044 & 0.044 & 0.043 & 0.060 & \\
\hline 8. Entorhipidium tenue (JQ956544) & 0.056 & 0.056 & 0.056 & 0.056 & 0.043 & 0.054 & 0.043 \\
\hline
\end{tabular}

Tabla 3. Estimaciones de distancias entre secuencias de especies del Orden Philasterida, comparadas utilizando el marcador $\beta$-tubulina, basado en el número de sustituciones por sitio entre secuencias, con el modelo K2P.

\begin{tabular}{|c|c|c|c|c|c|c|c|c|}
\hline & Cepa (Número de accesión) & 1 & 2 & 3 & 4 & 5 & 6 & 7 \\
\hline 1. & CEPA 1PERU & & & & & & & \\
\hline 2. & Philasterides dicentrarchi (GQ342956) & 0.008 & & & & & & \\
\hline 3. & Euplotes focardii (FJ664406) & 0.155 & 0.162 & & & & & \\
\hline 4. & Paramecium tetraurelia (XM001433469) & 0.150 & 0.150 & 0.167 & & & & \\
\hline 5. & Tetrahymena thermophila (XM001025231) & 0.157 & 0.157 & 0.210 & 0.201 & & & \\
\hline 6. & Tetrahymena pyriformis (X12769) & 0.164 & 0.164 & 0.202 & 0.194 & 0.039 & & \\
\hline 7. & Sterkiella histriomuscorum (AY116145) & 0.220 & 0.220 & 0.182 & 0.187 & 0.161 & 0.140 & \\
\hline 8. & Caenorhabditis briggsae (XM002643430) & 0.254 & 0.266 & 0.293 & 0.314 & 0.270 & 0.287 & 0.299 \\
\hline
\end{tabular}

Tabla 2. Estimaciones de divergencia entre secuencias de especies del Phylum Ciliophora comparadas utilizando el marcador $\mathrm{COI}$, basado en el número de sustituciones por sitio entre secuencias, con el modelo K2P.

\begin{tabular}{|c|c|c|c|c|c|c|c|c|c|c|c|c|c|}
\hline & Cepa (Número de accesión) & 1 & 2 & 3 & 4 & 5 & 6 & 7 & 8 & 9 & 10 & 11 & 12 \\
\hline 1 & CEPA 1PERU & & & & & & & & & & & & \\
\hline 2 & Miamiensis avidus (EU831233) & 0.001 & & & & & & & & & & & \\
\hline 3 & Miamiensis avidus (EU831213) & 0.018 & 0.017 & & & & & & & & & & \\
\hline 4 & Miamiensis avidus (EU831218) & 0.059 & 0.057 & 0.060 & & & & & & & & & \\
\hline 5 & Philasterides dicentrarchi (GQ342957) & 0.066 & 0.064 & 0.058 & 0.107 & & & & & & & & \\
\hline 6 & Miamiensis sp (CBOLN008-07) & 0.018 & 0.017 & 0.014 & 0.062 & 0.061 & & & & & & & \\
\hline 7 & Miamiensis sp (CBOLN007-07) & 0.021 & 0.020 & 0.014 & 0.065 & 0.046 & 0.017 & & & & & & \\
\hline 8 & Miamiensis sp (CBOLN006-7) & 0.059 & 0.057 & 0.059 & 0.010 & 0.107 & 0.060 & 0.063 & & & & & \\
\hline 9 & Cyclidium sp(FJ905128) & 0.255 & 0.253 & 0.257 & 0.230 & 0.312 & 0.269 & 0.265 & 0.228 & & & & \\
\hline 10 & Tetrahymena sp(EF070320) & 0.271 & 0.269 & 0.279 & 0.277 & 0.333 & 0.281 & 0.275 & 0.273 & 0.287 & & & \\
\hline 11 & Tetrahymena rostrata (EF070305) & 0.286 & 0.283 & 0.285 & 0.279 & 0.356 & 0.298 & 0.296 & 0.283 & 0.288 & 0.101 & & \\
\hline 12 & Tetrahymena tropicalis (GU439306) & 0.289 & 0.287 & 0.289 & 0.281 & 0.353 & 0.299 & 0.295 & 0.275 & 0.269 & 0.094 & 0.089 & \\
\hline 13 & Tetrahymena canadensis (GU439208) & 0.287 & 0.285 & 0.287 & 0.285 & 0.360 & 0.300 & 0.298 & 0.285 & 0.290 & 0.105 & 0.008 & 0.087 \\
\hline
\end{tabular}

clado a $M$. avidus y $P$. dicentrarchi, con un $100 \%$ de robustez, separándolo de otros géneros como Tetrahymena y Cyclidium.

A pesar que para $\beta$-tubulina se encontraron pocas secuencias disponibles en el GenBank, sin embargo se pudo identificar a la cepa 1PERU como semejante a $P$. dicentrarchi GQ342956 en un 99\%, presentando menores valores de similitud con Euplotes focardii FJ664406 (91\%), Paramecium tetraurelia XM_001433469 (87\%) y T. thermophila XM_001025231 (86\%). Se observaron 3 sustituciones al compararla con GQ342956, siendo dos de ellas transiciones en las posiciones nucleotídicas 48 y 204, y una transversión en 345. Valores de divergencias menores a 0.1 fueron observados entre las secuencias 1PERU y otra de la misma especie, y mayores a 0.15 respecto a otros géneros (Tabla 3). El agrupamiento mostró la formación de un clado con $P$. dicentrarchi, con un $100 \%$ de robustez.

Observaciones morfológicas e histológicas.- Los ciliados se observaron muy activos en las muestras en fresco, con pro- medios de $45.8 \mu \mathrm{m}$ de largo, $21.1 \mu \mathrm{m}$ de ancho y $10.3 \mu \mathrm{m}$ de longitud del cilio caudal, (medidas en base a una muestra de 100 ciliados). Se observaron formas elongadas piriformes, de extremo caudal redondeado y extremo anterior puntiagudo. Presencia de abundante ciliatura somática, con un cilio caudal más grande que los de la ciliatura somática. Se apreció además el aparato bucal, la vacuola contráctil y el poro de la vacuola contráctil (Fig. 3).

A nivel histológico, se observó una severa infección de ciliados hallados en casi todos los órganos internos como bazo, intestino, hígado, riñón, cerebro, corazón, branquias, cerebro y músculo.

No se observaron ciliados en el interior del bazo, pero sí masas de estos conteniendo restos de células en el tejido periesplénico conformado por tejido pancreático necrosado (Fig. 4A); además se evidenció la cápsula esplénica parcialmente desprendida del parénquima. 

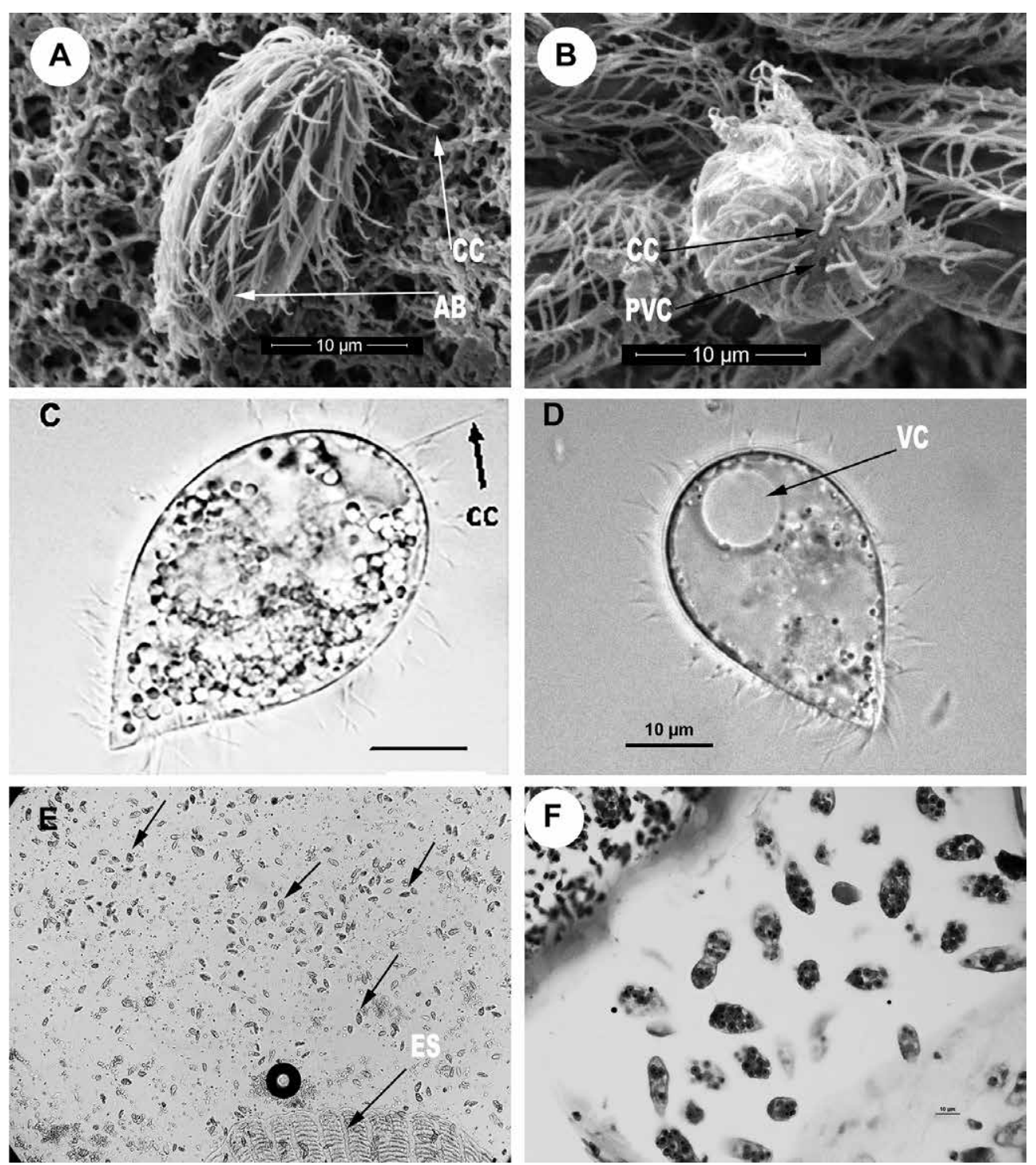

Figura 3. (A) y (B) Microfotografía electrónica de barrido (SEM) mostrando la ciliatura del protozoario ciliado $M$. avidus (Sin. P. dicentrarchi). Se aprecia el cilio caudal (CC), la ciliatura somática, el aparato bucal (AB) y el poro de la vacuola contráctil (PVC) en el extremo caudal. (C) y (D) Montaje en fresco bajo microscopio compuesto. Se aprecia algunas características del ciliado, además de la vacuola contráctil (VC). (E) Montaje en fresco al microscopio compuesto. Vista panorámica de raspado de piel de lenguado $P$. adspersus con escuticociliatosis. Se aprecia un número importante de ciliados (flechas) y restos de tejido, como escamas (ES), sobre la lámina (10X). (F) Ciliados conteniendo eritrocitos en su citoplasma (tinción H\&E). Barra de escala $=10 \mu \mathrm{m}$.

A nivel del tejido intestinal, se observó el desprendimiento parcial de la mucosa conjuntamente con necrosis de las microvellosidades del epitelio intestinal (Fig. 4B). Además, se evidenciaron numerosos ciliados en la lámina propia del tracto gastrointestinal de los peces infectados ocasionando una severa infiltración de células mononucleares del sistema inmunológico.

En el hígado, ocasionalmente se observaron ciliados entre los hepatocitos causando necrosis difusa del parénquima hepático; los ciliados fueron observados en más abundancia en el tejido pancreático del hígado rodeando las venas centro lobulillares, las cuales se encontraron congestionadas y con infiltración leucocitaria (Fig. 4C), alrededor de conductos biliares y vasos sanguíneos.

Por otro lado, se observó la presencia de numerosos ciliados y una severa reacción inflamatoria debajo de la cápsula renal, además de numerosos linfocitos y neutrófilos (Fig. 4D). En el parénquima renal se observaron células hematopoyéticas, melanomacrófagos y numerosos túbulos renales tapizadas de células epiteliales edematizadas. No se apreciaron ciliados en el parénquima renal.

En tejido cardíaco, se observó escasa afectación de los ciliados, generalmente con presencia sobre el epicardio y entre el epicardio y miocardio (Fig. 5A), causando un engrosamiento y desprendimiento del epicardio.

Las branquias presentaron necrosis del tejido epitelial, hiperplasia, y fusión lamelar; los ciliados solamente fueron evidenciados en la vasculatura interna de las lamelas primarias y secundarias (Fig. 5B).

El cerebro fue el órgano más parasitado por ciliados, encontrándose infectado en la mayoría de peces analizados. Se observó licuefacción del tejido, infiltración leucocitaria y masas de ciliados reemplazando las células nerviosas y en la periferia del parénquima cefálico causando encefalitis (Fig. 5C).

Lesiones necróticas, fascículos musculares lisados y grandes masas de ciliados fueron las características del músculo esquelético debajo de las capas de la epidermis. Se observó también, una cantidad importante de ciliados conteniendo numerosos eritrocitos en el citoplasma (Fig. 3F y 5D), además de severa infiltración leucocitaria y ciliados entre los radios cartilaginosos de las aletas.

\section{Discusión}

El lenguado $P$. adspersus está incluido entre las especies prioritarias para el desarrollo de la acuicultura marina del Perú, sin 

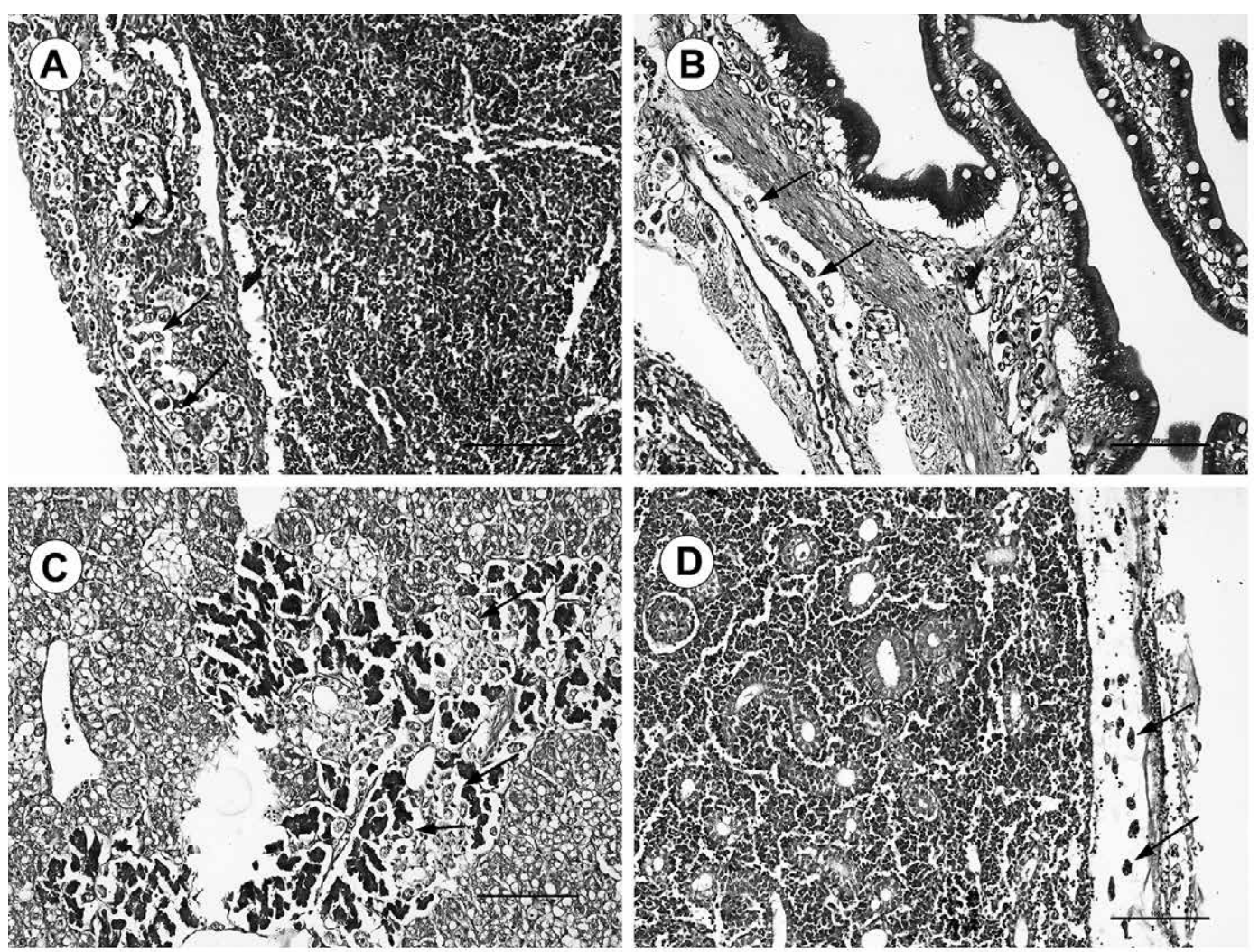

Figura 4. Cortes histológicos de tejidos de $P$. adspersus infectados por el ciliado $M$. avidus. (A) Infección de ciliados en tejido pancreático que rodea el bazo. (B) Invasión de ciliados en el tracto digestivo, se observan en la serosa, muscular y lamina propia. La mucosa se encuentra desprendida. (C) Numerosos ciliados causando necrosis del tejido pancreático y hepatocitos. (D) Reacción inflamatoria debido a la infección por ciliados debajo de la capa del parénquima renal. Tinción H\&E. Barra = $100 \mu \mathrm{m}$.
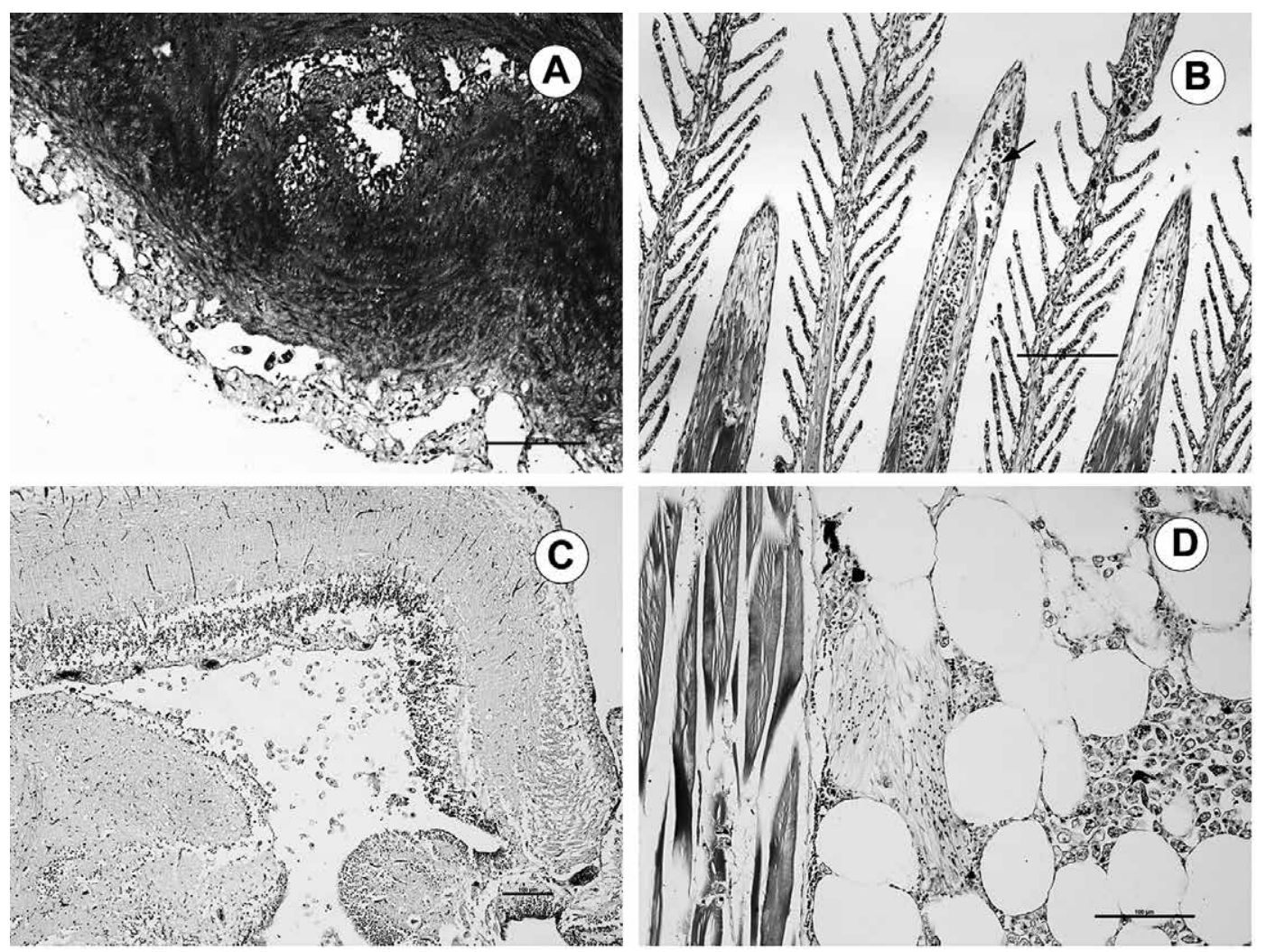

Figura 5. Cortes histológicos de tejidos de $P$. adspersus infectados por el ciliado $M$. avidus. (A) Ciliados entre el epicardio y miocardio. Se aprecia el epicardio engrosado y desprendido del miocardio. (B) Ciliados entre la vasculatura de una lamela primaria. (C) Cerebro de P.adspersus invadida de parásitos. Se aprecia licuefacción de las células nerviosas. (D) Fibras musculares invadidas de parásitos y el resto en proceso de lisis. Tinción H\&E. Barra de escala $100 \mu \mathrm{m}$. 
embargo se conoce poco sobre las enfermedades relacionadas a esta especie mantenida en cautiverio en nuestro país.

Los signos clínicos externos hallados en este estudio coinciden con lo reportado por otros autores para la enfermedad en peces planos (Yoshinaga \& Nakazoe 1993, Dyková \& Figueras 1994, Sterud et al. 2000, Iglesias et al. 2001, Jee et al. 2001, Kim et al. 2004) a excepción de la exoftalmia que no se evidenció en $P$. adspersus, en contraste con reportado para otros peces (Iglesias et al. 2001, Paramá et al. 2003). Algunos de los signos externos más evidentes en $P$. adspersus con escuticociliatosis fueron el abultamiento en la cavidad visceral con acumulación de líquido ascítico conteniendo abundante cantidad de ciliados, además de lesiones necróticas del epitelio a modo de manchas grises sobre el lado ocular en ejemplares infectados.

El uso de los marcadores $18 \mathrm{~S}$, COI y $\beta$-tubulina permitieron identificar a la cepa como $M$. avidus, mostrando una sinonimia con $P$. dicentrarchi. Song y Wilbert (2000) y Song et al. (2009) consideran a $M$. avidus ser un sinónimo senior de $P$. dicentrarchi.

Los análisis utilizando la región $18 \mathrm{~S}$ permitieron discriminar a la cepa en estudio del género Philasterides. Kim et al. (2004) identificaron al ciliado $P$. dicentrarchi aislado de $P$. olivaceus en Korea, utilizando la región ARNr SSU; Jung et al. (2005) también reportaron la presencia del parásito describiéndolo como $M$. avidus causante de escuticociliatosis para la misma especie. Posteriormente, Paramá et al. (2006) reportaron la posible sinonimia de $P$. dicentrarchi con $M$. avidus mediante análisis de $390 \mathrm{pb}$ de la región SSU, que fue confirmado posteriormente por Jung et al. (2007). Song et al. (2009) identificaron cepas de $M$. avidus utilizando secuencias SSU aisladas de 4 especies diferentes de lenguados, y caracterizándolas además con diferencias antigénicas entre ellas.

En la actualidad, secuencias de la región ribosomal $18 \mathrm{~S}$ son muy utilizadas para inferir relaciones evolutivas entre los ciliados, mostrando algunas discrepancias con los estudios morfológicos. El nivel de divergencia observado al comparar la secuencia $18 \mathrm{~S}$ con las obtenidas en otros estudios ( $0 \%$ entre secuencias de la misma especie, y $4.9 \%$ respecto a $P$. armatalis), corrobora el alto nivel de conservación del gen para este grupo. De igual forma Jung et al. (2011) reportaron un alto nivel de conservación del SSU para $M$. avidus, con una alta homología con $P$. dicentrarchi (99.83\%), resultando útil para estudios de identificación de especies de este grupo.

Por otro lado, las divergencias reportadas en este estudio entre secuencias de la misma especie utilizando el marcador COI (6.6\%) fueron mayores a las registradas con 18S. Jung et al. (2011) reportaron la presencia de variaciones a nivel intraespecífico (5.67\%), permitiendo establecer una diferenciación por serotipo de cepas. En ese sentido, Budiño Rodríguez (2011) resalta la utilidad de marcadores como COI y $\beta$-tubulina para detectar predominantemente variaciones intraespecíficas.

A pesar que no se cuentan con muchos datos de secuencias de $\beta$-tubulina para este grupo, sin embargo si se observó una diferenciación de $M$. avidus respecto a otros géneros, aunque con valores de divergencia entre especies y género entre 1 a $1.5 \%$, respectivamente. Si bien el polimorfismo del gen $\beta$-tubulina ha sido propuesto para ser utilizado en la identificación de especies, otros autores han reportado obtener genealogías inconsistentes al utilizar este marcador respecto a las basadas en genes ribo- somales o la ultraestructura. En tal sentido, resulta importante considerar el uso de múltiples marcadores, tanto nucleares como mitocondriales, para la caracterización de posibles diferencias intra e interespecíficas de cepas aisladas en futuros brotes que se registren en el Perú.

Es probable que el manejo de las condiciones del cultivo, factores físico químicos como la temperatura, contribuiría con proporcionar las condiciones para una ocurrencia de escuticociliatosis en $P$. adspersus en cautiverio. Este parámetro ambiental también ha sido relacionado con brotes de la enfermedad en otros peces y crustáceos (Cawthorn 1997, Munday et al. 1997, Iglesias et al. 2001, Azad 2007). Iglesias et al. (2003) reportaron una mayor tasa de crecimiento y reproducción de $P$. dicentrarchi, a $22{ }^{\circ} \mathrm{C}$ que a $15^{\circ} \mathrm{C}$ en cultivo. Puig et al. (2007) realizaron infecciones experimentales con $P$. dicentrarchi en el turbot $S$. maximus y reportaron que el curso de la infección fue rápido a mayor temperatura, concluyendo que este parámetro influye en el desarrollo de los parásitos.

Las lesiones necróticas en las aletas y piel de los ejemplares propiciaron la colonización de bacterias en $P$. adspersus infectados con $M$. avidus. En este estudio, se halló que la escuticociliatosis estuvo combinada con vibriosis producida por $V$. alginolyticus las cuales predominaron los cultivos microbiológicos, tal como lo reportado por Azad et al. (2007). Se ha reportado la vibriosis causada por $V$. alginolyticus y otros vibrios en lenguado $P$. adspersus en cautiverio (Miranda \& Rojas 1996), las cuales son bacterias ubicuas y oportunistas (Actis et al. 1999).

Severa necrosis del músculo esquelético, ciliados infectando la mayoría de órganos, principalmente el cerebro ocasionando encefalitis y con alta incidencia de necrosis vacuolativa del tejido entre otros cambios fueron los más frecuentes asociados a la infección con $M$. avidus en $P$. adspersus. Estos hallazgos histológicos están en concordancia con aquellos reportados en peces marinos como $S$. maximus (Jin et al. 2009) y P. olivaceous (Jung et al. 2007). En ese sentido, se ha reportado que los protozoarios producen proteasas las cuales destruyen tejidos (McKerrow 1989, North 1992, McKerrow et al. 1993, Zuo \& Woo 2000, Lee et al. 2003, Kwon et al. 2003). Probablemente algunas enzimas o alguna toxina de $M$. avidus destruyen la piel y músculo de $P$. adspersus, ocasionando úlceras y necrosis y posteriormente ganan entrada a los órganos internos por las microlesiones producidas y por vía sanguínea (Jin et al. 2009), tal como se ha hipotetizado (Paraná et al. 2003, Azad et al. 2007). Alvarez-Pellitero et al. (2004) reportaron que la infección experimental por inmersión con $P$. dicentrarchi sólo fue eficaz después de la abrasión de branquias y piel, ya que en centros de cultivo, los parásitos pueden sobrevivir en el fondo de los tanques ricos en nutrientes, y llegar a ser significativamente patogénicos solo cuando los peces desarrollan lesiones superficiales (Jin et al. 2009)

Por otro lado, el cerebro fue el órgano más parasitado y además, fue el que se encontró infectado sin que otros órganos lo estén en el mismo ejemplar muestreado. Es importante resaltar que los peces en los que se encontraron ciliados en el cerebro generalmente no presentaron abultamiento de la cavidad visceral y los órganos internos estuvieron levemente parasitados o no parasitados. En ese sentido, Munday et al. (1997), sugirieron que la vía de infección nasal infectaba principalmente al cerebro como órgano diana, por eso está bien documentado que el cerebro se encuentra vacuolado y licuado en escuticociliatosis (Munday et 
al. 1997, Iglesias et al. 2001, Deveney et al. 2005) y es porque estos ciliados tienen la capacidad de reproducirse en este órgano utilizando la ruta nasal por medio de los nervios olfatorios y ópticos (Munday et al. 1997, Sterud et al. 2000, Paraná et al. 2003) causando la muerte del animal posteriormente.

A nuestro entender, es la primera ocasión que se describe la enfermedad y la especie en el Perú. Sin embargo, tenemos comunicación de esta patología con sintomatología parecida y agente etiológico afín a las características de los ciliados descritos en la escuticociliatosis desde el año 2013 en lenguado $P$. adspersus en cautiverio asociado a elevadas mortalidades (comunicación personal). Considerando que no existe tratamiento efectivo para la enfermedad, se requiere mayor atención a las buenas prácticas de cultivo de esta especie.

En conclusión, la mortalidad de $P$. adspersus en cautiverio en este trabajo puede ser atribuida a $M$. avidus (Sin. P. dicentrarchi) el cual es altamente invasivo acompañado de infecciones secundarias de origen bacteriano.

\section{Literatura citada}

Actis L.A., M.E. Tolmasky \& J.H. Crosa. 1999. Vibriosis, in: R.M. Stevenson \& P.T. Woo (Ed.), Fish Diseases and Disorders: Viral, Bacterial and Fungal Infections, Vol. 3. CAB International Publishing. Wallingford, UK. pp. 523-557. http:// dx.doi.org/10.1079/9781845935542.0000

Acuña E. \& L. Cid. 1995. On the ecology of two sympatric flounder of the genus Paralichthys in the Bay of Coquimbo, Chile. Neth. J. Sea Res. 34: 1-11. http://dx.doi.org/10.1016/00777579(95)90011-X

Alvarez-Pellitero P., O. Palenzuela, F. Padrós, et al. 2004. Histophagous scuticociliatids (Ciliophora) parasitizing turbot Scophthalmus maximus: morphology, in vitro culture and virulence. Folia Parasitologica 51:177-187.

Azad I., A. Al-Marzouk, C. James, S. et al. 2007. Scuticociliatosisassociated mortalities and histopathology of natural infection in cultured silver pomfret (Pampus argenteus Euphrasen) in Kuwait. Aquaculture 262:202-210. http://dx.doi. org/10.1016/j.aquaculture.2006.10.03 3.

Budiño Rodríguez B. 2011. Caracterización de la variabilidad intraespecífica en Philasterides dicentrarchi, agente etiológico de la escuticoliatosis del rodaballo en cultivo. Memoria, Doctor en Biología. Universidad de Santiago de Compostela. http:// hdl.handle.net/10347/3689

Cawthorn R.J. 1997. Overview of Bumper car disease impact on the North American lobster fishery. Int. J. Parasitol. 27:167172. http://dx.doi.org/10.1016/S0020-7519 (96)00146-4

Cheung P., R. Nigrelli, G. Ruggieri.1980. Studies on the morphology of Uronema marinum Dujardin (Ciliatea: Uronematidae) with a description of the histopathology of the infection in marine fishes. J. Fish. Dis. 3:295-303. http://dx.doi. org/10:10.1111/j.1365-2761.1980.tb00400.x.

Deveney M., T. Bayly, C. Johnston, et al. 2005. A parasite survey of farmed Southern bluefin tuna, Thunnus maccoyii (Castelnau). J. Fish Dis. 28, 279-284. http://dx.doi.org/ doi:10.1111/j.1365-2761.2005.00629.x

Dragesco A., J. Dragesco, F. Coste, et al. 1995. Philasterides dicentrarchi, n. sp., (ciliophora, scuticociliatida), a histophagous opportunistic parasite of Dicentrarchus labrax (Linnaeus, 1758), a reared marine fish. European Journal of Protistology. 31; 3: 327-340. http://dx.doi.org/doi:10.1016/ S0932-4739 (11)80097-0

Dyková I. \& A. Figueras.1994. Histopathological changes in turbot Scophthalmus maximus due to a histophagous ciliate. Dis. Aquat. Org., 18: 5-9. http://dx.doi.org/doi: 10.3354/ dao076131

Elston R.A., D. Cheney, P. Frelier \& D. Lynn.1999. Invasive orchitophryid ciliate infections in juvenile Pacific and Kumomoto oysters, Crassostrea gigas and Crassostrea sikamea. Aquaculture 174:1-14. http://dx.doi.org/10.1016/S0044-8486 (98)00512-2
Gill, P. \& R. Calinan. 1997. Ulcerative dermatitis associated with Uronema sp. infection of farmed sand whiting Sillago ciliate. Australian Veterinary Journal. 75:357. http://dx.doi. org/10:10.1111/j.1751-0813.1997.tb15714.x.

Harikrishnan R., S. Balasundaram, \& M. Heo. 2010. Scuticociliatosis and its recent prophylactic measures in aquaculture with special reference to South Korea: Taxonomy, diversity and diagnosis of scuticociliatosis: Part I Control strategies of scuticociliatosis: Part II. Fish \& Shellfish Immunology. 29, 1:15-31. http://dx.doi.org/doi:10.1016/j.fsi. 2010. 02.026

Iglesias R., A. Paramá, M. Alvarez, et al. 2003. In vitro growth requirements for the fish pathogen Philasterides dicentrarchi (Ciliophora, Scuticociliatida). Vet. Parasitol.20; 111(1):1930. http://dx.doi.org/10.1016/S0304-4017(02)00350-3.

Iglesias R., A. Paraná, F. Alvarez, et al. 2001. Philasterides dicentrarchi (Ciliophora, Scuticociliatida) as the causative agent of scuticociliatosis in farmed turbot Scophthalmus maximus in Galicia (NW Spain). Dis. Aquat. Org. 46: 47-55. http:// dx.doi.org/doi:10.3354/dao046047

Jee B.Y., Y.C. Kim \& M.S. Park. 2001. Morphology and biology of parasite responsible for scuticociliatosis of cultured olive flounder Paralichthys olivaceus. Dis. Aquat. Org., 47: 49-55. http://dx.doi.org/doi:10.3354/dao047049

Jin C., R. Harikrishnan, Y. Moon, et al. 2009. Histopathological changes of Korea cultured olive flounder, Paralichthys olivaceus due to scuticociliatosis caused by histophagous scuticociliate, Philasterides dicentrarachi. Vet. Parasitol. 161 (3):292-301. http://dx.doi.org/doi:10.1016/j.vetpar.2009.01.033

Kim S., J. Cho, E. Lee, et al.2004. Pseudocohnilembus persalinus (Ciliophora: Scuticociliatida) is an additional species causing scuticociliatosis in olive flounder Paralichthys olivaceus. Dis. Aquat. Org. 62: 239-244. http://dx.doi.org/doi:10.3354/ dao062239

Kim S., J. Cho, S. Kim, et al. 2004. Occurrence of scuticociliatosis in olive flounder Paralichthys olivaceus by Philasterides dicentrarchi (Ciliophora: scuticociliatida). Dis. Aquat. Org. 62(3): 233-238. http://dx.doi.org/doi:10.3354/ dao062233

Kwon S., C. Kim \& K. Kim. 2003. Differences between short and long-term cultures of Uronema marinum (Ciliophora: Scuticociliatida) in chemiluminescence inhibitory activity, antioxidative enzyme and protease activity. Aquaculture, 221: 107-114. http://dx.doi.org/doi:10.1016/S0044-8486 (03)00114-5

Miranda R.C. \& R. Rojas. 1996. Vibriosis en el lenguado Paralichthys adspersus (Steindachner 1897) en cautiverio. Rev.Biol.Mar. Valparaíso. 31(1):1-9

Moustafa M., N. Tange, A. Shimada, et al. 2010. Experimental scuticociliatosis in Japanese flounder (Paralichthys olivaceus) infected with Miamiensis avidus: pathological study on the possible neural routes of invasion and dissemination of the scuticociliate inside the fish body. J. Vet. Med. Sci. 72(12):1557-63

Munday B., P. O’Donoghue, M.Watts, et al. 1997. Fatal encephalitis due to the scuticociliate Uronema nigricans in sea-cage, southern bluefin tuna Thunnus maccoyii. Dis. Aquat. Org., 30:17-25. http://dx.doi.org/doi: 10.3354/dao030017

Paramá A., R. Iglesias, M.F. Alvares., et al. 2003. Philastherides dicentrarchi (Ciliphora: Scuticociliatida) experimental infecction and posible routes of entry in farmed turbot (Scophthalmus maximus). Aquaculture http://dx.doi.org/doi:217:73-80. 10.1016/S0044-8486(02)00523-9

Pequeño G. 1989. Peces de Chile. Lista sistemática revisada y comentada. Rev. Biol. Mar. Valparaíso. 24(2): 1-132.

Piazzon M.E., J. Leiro J. \& J. Lamas. 2013. Fish immunity to scuticociliate parasites. Developmental \& Comparative Immunology. 41: 248-256. http://dx.doi.org/10:10.1016/j. dci.2013.05.022.

PRODUCE. 2013. (en línea). Programa Nacional de Ciencia, Desarrollo Tecnológico e Innovación en Acuicultura $(\mathrm{C}+\mathrm{DT}+\mathrm{i})$ 2013-2021. Acceso 25/05/2016.

Puig L., R. Traveset, O. Palenzuela, et al. 2007.Histopathology of experimental scuticociliatosis in turbot Scophthalmus maximus Dis. Aqua. Org. Vol. 76: 131-140. http://dx.doi.org/doi: $10.3354 /$ dao076131. 
Rossteuscher S., C. Wenker, T. Jermann, et al.2008. Severe scuticociliate (Philasterides dicentrarchi) infection in a population of sea dragons (Phycodurus eques and Phyllopteryx taeniolatus). Vet.Pathol.45:546- 550. http://dx.doi.org/10:10.1354/ vp.45-4-546.

Sanmartín M., A. Paraná, R. Castro, et al. 2008. Vaccination of turbot, Psetta maxima, against the protozoan parasite Philasterides dicentrarchi: effects on antibody production and protection. Journal of Fish Diseases. 1, 135-140. http://dx.doi. org/10.1111/j.1365-2761.2007.00876.x

Scholz T. 1999. Parasites in cultured and feralfish. Vet. Para. 84:317335. http://dx.doi.org/10.1016/S0304-4017(99)00039-4

Siefeld W., M. Vargas \& I. Kong. 2003. Primer registro de Etropus edenes Jordán, 1889, Bothus constellatus Jordán \& Goss, 1889, Achirus klunzingeri (Steindachner, 1880) y Symphurus elongatus (Gunther, 1868) (Pisces, Pleuronectiformes) en Chile, con comentarios sobre la distribución de los lenguados chilenos. Invest. Mar. Valparaíso, 31: 51-65. http://dx.doi. org/10.4067/S0717-71782003000100006

Silva A. \& M. Oliva. 2010. Revisión sobre aspectos biológicos y de cultivo del lenguado chileno (Paralichthys adspersus). Lat. Am. Jor. of Aqua. Res. 38(3), 377-386. http://dx.doi. org/10.4067/S0718-560X2010000300003
Song J., K. Sasaki, T. Okada, et al.2009. Antigenic differences of the scuticociliate Miamiensis avidus from Japan. J.Fish. Dis.32:1027-1034. http://dx.doi.org/doi:10.1111/j.13652761.2009.01089.x.

Sterud E., M. Hansen \& T.A. M. 2000. Systemic infection with Uronema-like ciliates in farmed turbot, Scophthalmus maximus. J. Fish. Dis. 23:33-37.http://dx.doi.org/doi:10.1046/j.13652761.2000.00204.x

Stidworthy M., M. Garner, D. Bradway, B. et al. 2013. Systemic Scuticociliatosis (Philasterides dicentrarchi) in Sharks, Vet. Patho. 00(0):1-5. http://dx.doi. org/10:10.1177/0300985813492800

Thompson J. \& L. Moewus.1964. Miamiensis avidus n. g., n. sp., a marine facultative parasite in the ciliate Order Hymenostomatida. J. Protozool., 11: 378-381. http://dx.doi. org/10:10.1111/j.1550-7408.1964.tb01766.x

Yoshinaga T. \& J. Nakazoe. 1993. Isolation and in vitro cultivation of an unidentified ciliate causing scuticociliatosis in Japanese flounder (Paralichthys olivaceus). Fish Pathol 28:131-134 